\title{
Point Spread Function analysis, modeling, simulations and laboratory testing for TMT NFIRAOS
}

\author{
L. Gilles ${ }^{a}$, L. Wanga, E. Mieda ${ }^{b}$, J.P. Véran ${ }^{\mathrm{b}}$ and C. Boyer ${ }^{\mathrm{a}}$
}

aThirty Meter Telescope Observatory Corp., 100 West Walnut Street, Suite 300, Pasadena, CA

91124, USA; bNRC Herzberg Astronomy and Astrophysics, 5071 West Saanich Road, Victoria, British Columbia V9E 2E7, Canada

\begin{abstract}
This paper discusses four on-axis natural guide star adaptive optics (NGS AO) point spread function reconstruction (PSFR) algorithms developed and benchmarked on simulated and laboratory telemetry data. Simulated telemetry was provided by the MAOS software configured to simulate the Thirty Meter Telescope (TMT) NFIRAOS system, or the Herzberg NFIRAOS Optical Simulator (HeNOS) bench. Laboratory data was acquired on the HeNOS bench at the Institute of Astrophysics at the National Research Council of Canada.
\end{abstract}

Keywords: Adaptive optics, point spread function reconstruction

\section{INTRODUCTION}

Point spread function (PSF) knowledge is critical for any adaptive optics (AO) astronomical science program aiming at obtaining high angular resolution information. Examples of such programs include photometry and astrometry in crowded and sparse stellar fields, detection and characterization of exoplanets, determination of precision orbits at the Galactic Center, etc. [1][2][3][[4]. In order to enable such science programs, AO systems on existing telescopes and future extremely large telescopes are required to meet tight photometry and astrometry budgets. For instance, the adaptive optics system under design for the Thirty Meter Telescope (TMT) project is required to provide $2 \%$ differential photometry (relative brightness between two point sources) over a 30" diameter field of view (FoV) for a 10 min exposure at a wavelength of 1 micron, and 50 microarcsec root-mean-square (RMS) time dependent differential astrometry (relative separation between two point sources) over the same FoV for a $100 \mathrm{sec}$ exposure in $\mathrm{H}$ band [5].

Véran pioneered AO point spread function reconstruction (PSFR) three decades ago [6], by developing an algorithm to reconstruct the on-axis long exposure PSF of a bright natural guide star (NGS) from the wavefront sensor (WFS) measurement covariance matrix accumulated during the science exposure. Ten years later, Britton [7] and Flicker [8] extended Véran's algorithm to include the effects of anisoplanatism, which is an effect arising when the guide star used to close the AO loop is at a different range and/or angular position than the science target. Progress implementing the anisoplanatic PSFR algorithm has been reported recently by the Keck observatory [9].

In this paper, we are not concerned with anisoplanatism, the guide star and science target are both on-axis and at infinity. Anisoplantism will be addressed in a following study focusing on tomographic PSFR. The manuscript is organized as follows: Section 2 describes the on-axis NGS AO PSFR algorithms investigated. Section 3 presents three sets of results: (1) results obtained from simulated NFIRAOS telemetry, (2) results obtained from simulated HeNOS telemetry, and (3) results obtained from actual HeNOS bench telemetry. Finally, Section 4 concludes the paper.

\section{NGS AO ON-AXIS PSFR ALGORITHMS}

\subsection{Overview}

The optical transfer function (OTF) is defined as the Fourier transform of a unit flux PSF. The long exposure OTF is estimated as the product of static and turbulence OTFs:

$$
\widehat{O T F}=O T F_{\mathrm{Stat}} \cdot \widehat{O T F}_{\mathrm{Tur}}
$$

It has been shown that decoupling static and residual turbulence holds to a high level of accuracy [10], hence the multiplicative approximation made in (2.1) is accurate. Since PSFs are recorded on digital sensors, all quantities in (2.1) 
are discrete, matching the Spatial Domain (SD) detector pixel sampling and field of view (FoV). In the SD, we denote by $\Delta \theta=\operatorname{atan}\left(h^{\prime} / f\right)$ the detector pixel angular size ( $h^{\prime}$ being the pixel linear size and $f$ the camera focal length) and by $n$ the 1-dimensional number of pixels of the recorded PSF. In the Fourier Domain (FD), the detector is characterized by its bandwidth (BW) and its frequency sampling, which are respectively given by $\mathrm{BW}=n \Delta u=1 / \Delta \theta=n / \mathrm{FoV}$ and $\Delta u=\lambda / \Delta x=1 / \mathrm{FoV}$, where $\lambda$ denotes the wavelength at which the OTF is recorded, and $\Delta x$ is the wavefront sampling associated to the detector FoV. In order to avoid aliasing issues, the detector should be at least Nyquist sampled, i.e. $\xi=\mathrm{BW} / \mathrm{BW}_{\mathrm{opt}}=\delta \theta /(2 \Delta \theta) \geq 1$ with $\delta \theta=\lambda / D, \mathrm{BW}_{\mathrm{opt}}=2 / \delta \theta$ denoting the system optical bandwidth and $D$ the telescope aperture diameter. In order to properly capture high-spatial frequencies in the estimated turbulence degraded PSF, estimation is done typically over a broader FoV given by $\mathrm{FoV}_{\mathrm{evl}}=n_{\mathrm{evl}} \Delta \theta=\lambda / \Delta x_{\mathrm{evl}} \gg \mathrm{FoV}$ than that of the recorded PSF. Note that the BW of the estimated OTF matches that of the recorded OTF, i.e. $\Delta \theta_{\text {evl }}=\Delta \theta$. We thus have:

$$
\widehat{O T F}_{\text {Tur }}=\mathcal{F}_{n \times n}\left\{\operatorname{Crop}\left[\widehat{P S F}_{\text {Tur,evl }}\left(\Delta \theta, n_{\text {evl }}\right), n\right]\right\}
$$

where the function "Crop" crops the $n_{\text {evl }} \times n_{\text {evl }}$ reconstructed PSF to the final $n \times n$ PSF, and $\mathcal{F}_{n \times n}\{\cdot\}$ denotes the 2D $n \times n$ Fourier transform. Depending on whether actuator error or wavefront sensor (WFS) telemetry is used to reconstruct the turbulence degraded PSF, different error terms have to be taken into account in the processing. We are considering covariance and time history based algorithms, operating on either a time history of WFS gradients or a time history of deformable mirror (DM) actuator errors.

\subsection{Candidate Algorithms}

PSFR relies on the processing of AO system telemetry, supplemented by FD filters, pre-computed from simulation models. Those pre-computed filters are obtained from:

- A closed loop AO simulation for which only WFS aliasing and servo lag is present. This is achieved by (i) setting the location of the deformable mirror (DM) actuators to WFS subaperture vertices and modeling their influence using bilinear splines (the sensed $\mathrm{BW}, \lambda / d_{\mathrm{sa}}$, is therefore equal to the control $\mathrm{BW}, \lambda / d_{\mathrm{a}}$, where $d_{\text {sa }}$ denotes WFS subaperture size and $d_{\mathrm{a}}$ DM actuator pitch), (ii) projecting the simulated turbulence phase screen onto the orthogonal complement of the span of bilinear splines located at WFS subaperture vertices (the wavefront resulting from this projection is called the "unsensed wavefront"). The time history of the residual closed loop WFS gradients is used to compute in post-processing the gradient aliasing filter $K_{\text {ga }}$, whereas the science OTF is used to compute the science aliasing filter $K_{\text {sca }}$ and the phase screen projection is used to compute the open loop unsensed wavefront filter $K_{s}$.

- An open loop AO simulation without WFS to compute the uncorrectable wavefront error (WFE) (i.e. the DM fitting error) by projecting the simulated turbulence phase screen onto the orthogonal complement of the span of the DM actuator influence functions (IFs). The resulting filter will be denoted $K_{c}$.

We are considering the following 4 candidate algorithms:

$$
\widehat{O T F}_{\mathrm{Tur}, \mathrm{evl}}\left(\Delta u_{\mathrm{evl}}, n_{\mathrm{evl}}\right)=K_{\mathrm{sca}} \cdot\left\{\begin{array}{c}
\widehat{O T F}\left(C_{\mathrm{ea}}, C_{\mathrm{an}}\right) \\
\widehat{O T F}(\mathrm{ea}(t), \mathrm{an}(t)) \\
\widehat{O T F}(g(t), \mathrm{gn}(t)) \\
\widehat{O T F}\left(C_{g}, C_{\mathrm{gn}}\right)
\end{array}\right.
$$


where $C_{\mathrm{ea}}$ denotes the actuator error covariance matrix, $C_{\mathrm{an}}$ the actuator noise covariance matrix, ea $(t)$ the actuator error time history, an $(t)$ a realization of actuator error noise time history, $g(t)$ WFS gradient time history, gn $(t)$ a realization of WFS gradient noise time history, $C_{g}$ the WFS gradient covariance matrix, $C_{\mathrm{gn}}$ the WFS gradient noise covariance matrix, and $K_{\text {sca }}$ a common science aliasing filter pre-computed from a simulation model as described in the previous paragraph. Throughout this paper, we denote by $K \equiv O T F / O T F_{\mathrm{DL}}$ the OTF relative to the diffraction limited (DL) OTF.

- Algorithm1 reconstructs the turbulence degraded OTF using the actuator error and actuator noise covariance matrices. The structure function (SF) is computed from the covariance matrix and the long-exposure (LE) OTF is assembled from the SF. The algorithm can be expressed as follows:

$$
\widehat{O T F}\left(C_{\text {ea }}, C_{\text {an }}\right)=K_{c} \cdot \frac{U\left\{\widehat{O T F}\left(H_{a, \mathrm{e}} C_{\mathrm{ea}} H_{a, \mathrm{te}}^{T}\right)\right\}}{U\left\{\hat{K}\left(C_{\mathrm{an}}\right)\right\}}, \quad C_{\mathrm{an}}=H_{a, \mathrm{te}}\left[R C_{g \mathrm{n}} R^{T}\right] H_{a, \mathrm{te}}^{T}
$$

where $K_{c}$ is a filter accounting for uncorrectable (DM fitting) wavefront errors that is pre-computed from simulation, $U\{\cdot\}$ is the operator up-sampling from the telemetry BW to the evaluation BW, computed with bilinear splines sampled on the fine evaluation grid, $R$ denotes the control matrix running the AO loop and $H_{a \text {,te }}$ is an up-sampling operator for non-Fried geometries with DM actuators undersampling WFS subapertures, in which case $H_{a \text {, te }}$ upsamples using the DM actuator influence functions (IFs) from the actuator grid to a Fried geometry "telemetry" grid whose nodes are at WFS subaperture vertices. The telemetry grid is also used by the gradient-based algorithm to reconstruct the residual turbulence degraded wavefront and the covariance matrix. Finally, given a $q \times q$ covariance matrix $C$, the associated $q \times q$ SF is computed as follows:

$$
\mathrm{SF}=d \cdot 1^{T}+1 \cdot d^{T}-2 C
$$

where $d=\operatorname{diag}(C)$ is the $q \times 1$ vector formed by the diagonal entries of the covariance matrix $C$, and 1 is the $q \times 1$ vector of ones. The LE OTF at the $2 \mathrm{D}$ spatial frequency $u_{(j)}$ is given by:

$$
\operatorname{OTF}\left(u_{(j)}\right)=(\Delta x)^{2} \sum_{i} A\left(x_{(i)}\right) \cdot A\left(x_{(i)}+\lambda u_{(j)}\right) \cdot \exp \left[-\frac{k^{2}}{2} \mathrm{SF}\left(x_{(i)}, x_{(i)}+\lambda u_{(j)}\right)\right]
$$

where $k=2 \pi / \lambda, A\left(x_{(i)}\right)$ is the $i^{\text {th }}$ component of the telescope aperture amplitude vector, and $\operatorname{SF}\left(x_{(i)}, x_{\left(i^{\prime}\right)}\right)$ denotes row $i$ and column $i^{\prime}$ of the SF matrix defined in (2.5).

- Algorithm2 reconstructs the turbulence degraded OTF using the actuator error time history and realizations of the actuator noise time history. It can be expressed as follows:

$$
\widehat{O T F}(\mathrm{ea}(t), \operatorname{an}(t))=K_{c} \cdot \frac{\left\langle\operatorname{OTF}\left(H_{a, \mathrm{evl}} \mathrm{ea}(t)\right\rangle_{t}\right.}{\left\langle\hat{K}\left(H_{a, \mathrm{evl}} \mathrm{an}(t)\right\rangle_{t}\right.}, \quad \operatorname{an}(t)=R \cdot \operatorname{gn}(t), \quad \operatorname{gn}(t)=\left(C_{g \mathrm{n}}\right)^{1 / 2} \eta(t)
$$

where $H_{a \text {,evl }}$ is an up-sampling operator using the DM actuator IFs mapping actuator error onto the fine evaluation grid, $\eta(t)$ denotes a realization of zero-mean, unit covariance, normally distributed gradient noise, and $\langle\hat{K}\rangle_{t} \equiv\langle\widehat{O T F}\rangle_{t} / O T F_{\mathrm{DL}}$ with $\langle\widehat{O T F}\rangle_{t}=\mathcal{F}\left\{\langle\widehat{P S F}\rangle_{t}\right\}$ and $\widehat{P S F}=\left|\mathcal{F}_{n_{\mathrm{evl}} \times n_{\mathrm{evl}}}\left\{B \exp \left[i k \hat{\phi}\left(\Delta x_{\mathrm{evl}}, t\right)\right]\right\}\right|^{2}$ where $B$ denotes the 2D array computed by embedding the elements of the amplitude vector $A\left(x_{(i)}\right)$ in $(2.6)$ at the appropriate location, and $\hat{\phi}$ is the reconstructed aperture-plane phase array. 
- Algorithm3 reconstructs the turbulence degraded OTF from WFS gradient time history and realizations of WFS noise time history. It can be expressed as follows:

$$
\widehat{O T F}(g(t), g \mathrm{n}(t))=K_{s} \cdot K_{\mathrm{ga}} \cdot \frac{\left\langle\operatorname{OTF}\left(H_{\mathrm{te}, \mathrm{evl}} R_{\mathrm{te}} g(t)\right\rangle_{t}\right.}{\left\langle\hat{K}\left(H_{\mathrm{te}, \mathrm{evl}} R_{\mathrm{te}} g \mathrm{n}(t)\right\rangle_{t}\right.}
$$

where $K_{s}$ denotes the unsensed wavefront error filter, $K_{\mathrm{ga}}$ the WFS gradient aliasing filter, $R_{\mathrm{te}}$ the least-squares reconstructor (LSR) used to post-process gradients and reconstruct the wavefront on the telemetry grid, and $H_{\text {te,evl }}$ is an up-sampling operator using bilinear splines mapping the reconstructed wavefront to the finely sampled evaluation grid. $R_{\mathrm{te}}$ contains only global piston and global waffle in its null space, and it does not incorporate regularization, which is important to provide a faithful wavefront estimate from WFS gradients.

- Algorithm4 reconstructs the turbulence degraded OTF using the WFS gradient and gradient noise covariance matrices. The algorithm can be expressed as follows:

$$
\widehat{O T F}\left(C_{g}, C_{g \mathrm{n}}\right)=K_{s} \cdot K_{g a} \cdot \frac{U\left\{\widehat{O T F}\left(R_{\mathrm{te}} C_{g} R_{\mathrm{te}}^{T}\right)\right\}}{U\left\{\hat{K}\left(R_{\mathrm{te}} C_{g \mathrm{n}} R_{\mathrm{te}}^{T}\right)\right\}}
$$

Note that the above WFS gradient-based algorithms reduce to the actuator error based algorithms when DM actuators are located at WFS subaperture vertices (Fried geometry) and $R=R_{\mathrm{te}}$.

\section{SIMULATION RESULTS}

A minimum variance (MVR) control loop matrix tuned for 10mas RMS WFS noise per subaperture is used in all simulations, and all simulated wavefronts are finely sampled at a spatial resolution of $1 / 64 \mathrm{~m}$ to accurately capture highspatial frequencies and provide a finely sampled optical transfer function (OTF) (Fourier transform of the PSF), which is important for accurate performance evaluation.

\subsection{Performance metrics}

All four algorithms presented in Section 2.2 are compared in terms of their estimation error in Strehl ratio (SR), enclosed energy (EE), and PSF Profile i.e. spatial standard deviation (STD) of the PSF estimation error relative to the spatial STD of the measured/true PSF. Mathematically these three performance metrics are expressed as follows:

$$
\begin{aligned}
& \eta=\frac{\mathrm{SR}-\widehat{\mathrm{SR}}}{\mathrm{SR}}, \quad \epsilon(\Omega)=\frac{\mathrm{EE}(\mathrm{PSF}, \Omega)-\mathrm{EE}(\widehat{\mathrm{PSF}}, \Omega)}{\mathrm{EE}(\mathrm{PSF}, \Omega)}, \\
& \mathrm{EE}(\mathrm{PSF}, \Omega)=\frac{\Phi\{\mathrm{PSF}, \Omega\}}{\Phi\left\{\mathrm{PSF}, \Omega_{\text {max }}\right\}}, \quad \Omega \leq \Omega_{\text {max }}, \quad \Phi\{\mathrm{PSF}, \Omega\}=(\Delta \theta)^{2} \cdot \sum_{i, j \in \Omega} \mathrm{PSF}_{i j}, \\
& Q\left(\Omega_{\text {max }}\right)=\frac{\sqrt{\operatorname{Var}\{\mathrm{PSF}-\widehat{\mathrm{PSF}}\}}}{\sqrt{\operatorname{Var}\{\mathrm{PSF}\}}}=\frac{\sqrt{\mathrm{Mean}\left\{(\mathrm{PSF}-\widehat{\mathrm{PSF}})^{2}\right\}}}{\sqrt{\operatorname{Var}\{\mathrm{PSF}\}}}=\frac{\sqrt{\Phi\left\{(\mathrm{PSF}-\widehat{\mathrm{PSF}})^{2}, \Omega_{\text {max }}\right\}}}{\sqrt{\Phi\left\{(\mathrm{PSF}-\overline{\mathrm{PSF}})^{2}, \Omega_{\text {max }}\right\}}} \\
& \overline{\mathrm{PSF}}=\frac{\Phi\left\{\mathrm{PSF}, \Omega_{\text {max }}\right\}}{\Omega_{\text {max }}^{2}}=\frac{1}{N} \sum_{i, j \in \Omega_{\text {max }}} \mathrm{PSF}_{i j}, \quad \Phi\left\{\mathrm{PSF}, \Omega_{\text {max }}\right\}=\Phi\left\{\widehat{\left.\mathrm{PSF}, \Omega_{\text {max }}\right\}}\right.
\end{aligned}
$$

\subsection{Results obtained from simulated NFIRAOS telemetry}

NFIRAOS is the Thirty Meter Telescope (TMT) facility AO system [5]. The simulated TMT has a 30m-diameter pupil including 6 spiders and a central obscuration. The simulated NFIRAOS system consists of an order 60x60 ShackHartmann WFS and an order 60x60 pupil-conjugate DM with actuators registered to subaperture corners (Fried 
geometry) running in closed loop at $800 \mathrm{~Hz}$ frame rate with an integrator gain of 0.5 . The DM is modeled using bi-cubic IFs with $30 \%$ inter-actuator coupling (IAC) correcting frozen flow turbulence characterized by a $18.6 \mathrm{~cm}$ Fried parameter at half micron, a $30 \mathrm{~m}$ outer scale and a wind speed of $15 \mathrm{~m} / \mathrm{s}(34 \mathrm{~Hz}$ Greenwood frequency). The simulation records a Nyquist sampled on-axis 3840x3840 PSF at 2.2 micron (7.56mas pixels) exposed during 5,000 frames. A static loworder wavefront disturbance in the science path producing a $92 \%$ SR is included in the simulations. Finally, WFS noise is simulated at the level of 50mas RMS per subaperture.

Results for algorithms $2 \& 3$ (frame-by-frame PSFR) are shown in Figure 1, illustrating accurate reconstruction once all required filters are incorporated. Results for algorithms 1\&4 are shown in Figure 2, showing spurious OTF oscillations for algorithm 4 . These oscillations are triggered by local waflle modes present in the LSR post-processor and corrupting the SF. The oscillations damp out when oversampling is incorporated in the LSR post-processor, and they are not seen for the actuator telemetry covariance since the AO loop runs a MVR, which incorporates regularization. Figure 3 summarizes results for the most successful algorithms 1,2 and 3, which all yield SR and PSF Profile errors of only a few percent. Finally, Figure 4 illustrates similar results for the case of a simulation running on noise-free WFS gradients.

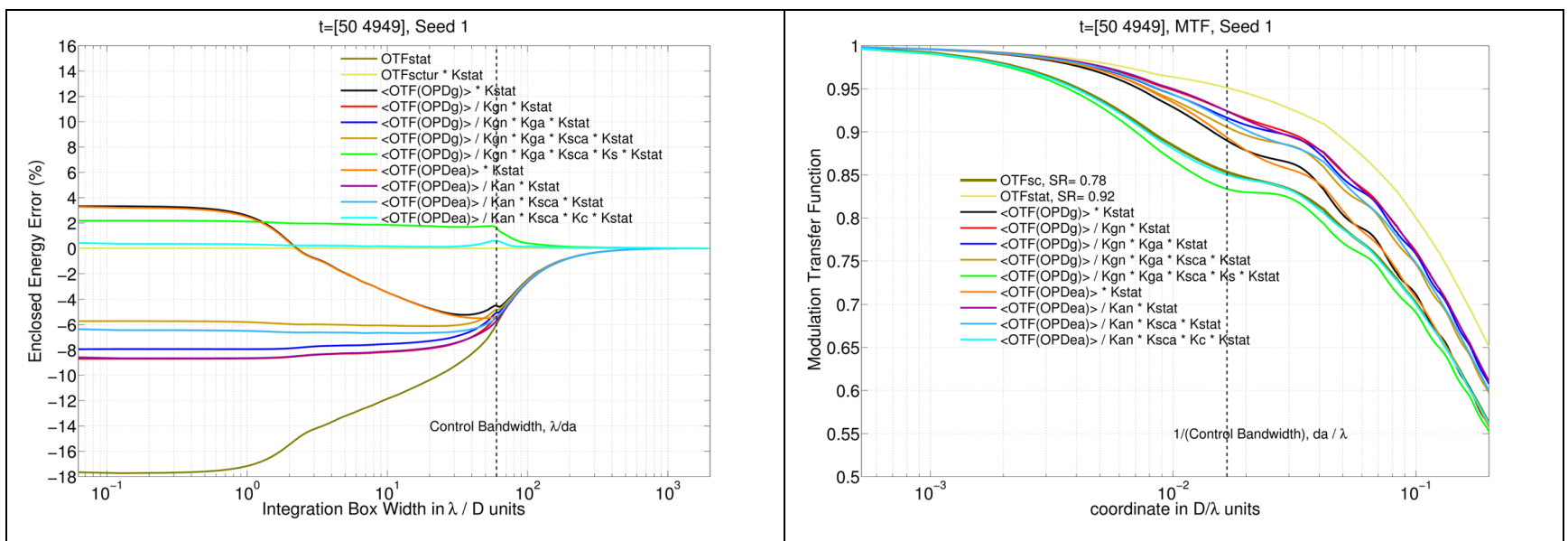

Figure 1: EE errors and OTF cross-sections for algorithms $2 \& 3$.
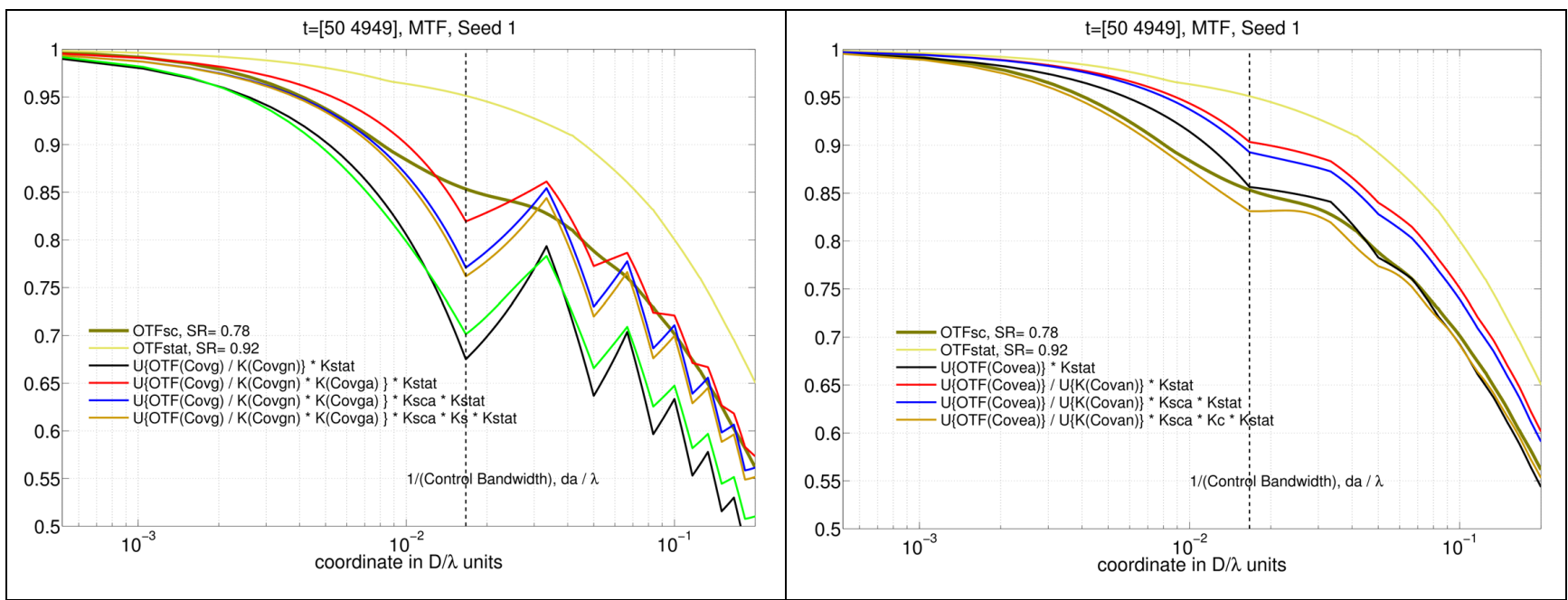

Figure 2: OTF cross-sections for algorithms $1 \& 4$. 

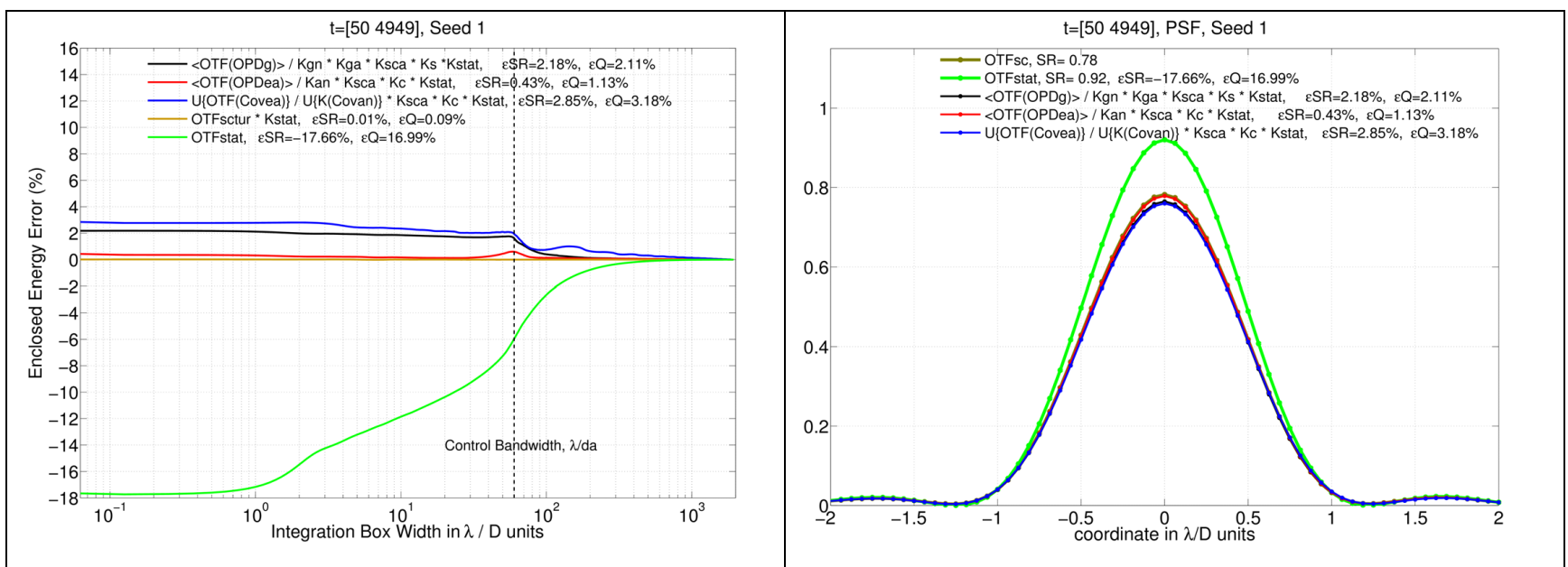

Figure 3: Left: EE errors for algorithms 3 (black), 2 (red), and 1 (blue). Right: reconstructed PSFs.
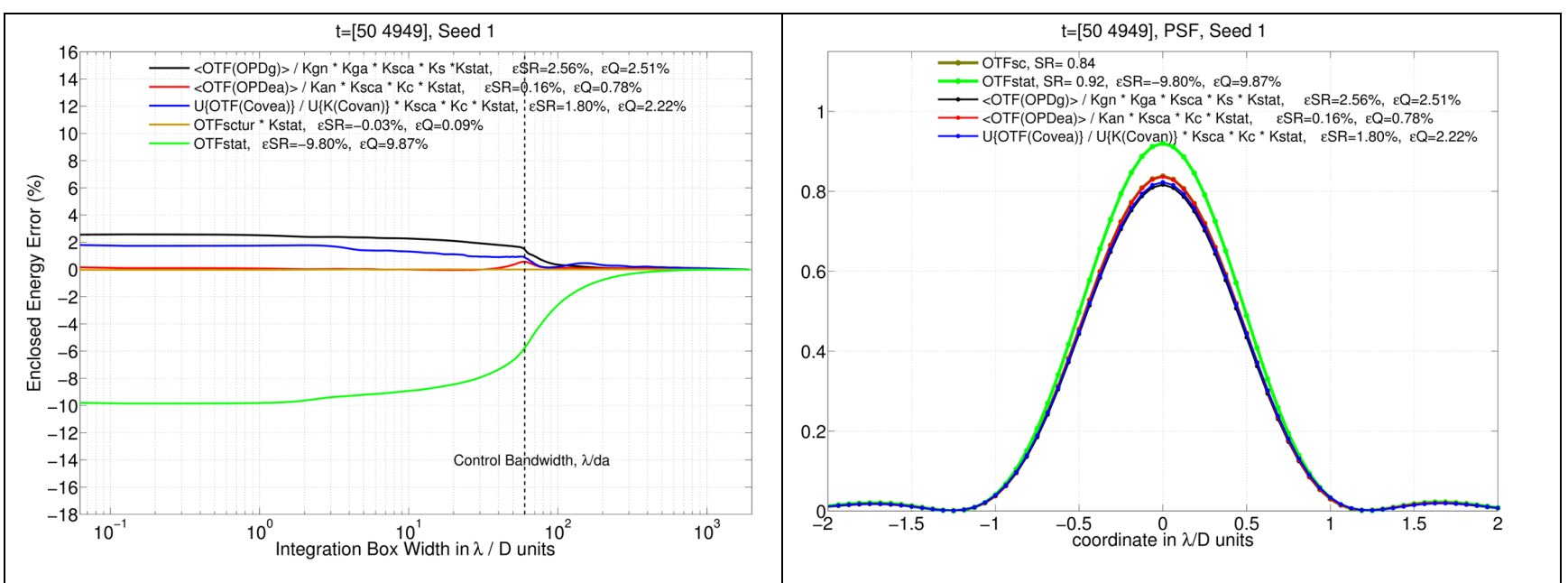

Figure 4: Same as Figure 3 but for a simulation excluding WFS noise.

\subsection{Results obtained from simulated HeNOS telemetry}

The HeNOS bench at NRC-Herzberg [11] simulates an $8.13 \mathrm{~m}$ telescope with a post-focal adaptive optics system consisting of an order 30x30 Shack-Hartmann WFS and an order 9x9 pupil-conjugate DM (non Fried geometry), running in closed loop with an integrator gain of 0.2. The DM IFs were simulated using bi-cubic splines with $41 \%$ IAC, and turbulence was simulated using a single pupil-conjugate phase screen producing a $58 \mathrm{~cm}$ Fried parameter at half micron, a $30 \mathrm{~m}$ outer scale and a wind speed of $15 \mathrm{~m} / \mathrm{s}(11 \mathrm{~Hz}$ Greenwood frequency). A science PSF was recorded at $670 \mathrm{~nm}$ with 5.6mas pixel sampling (Nyquist sampling is $8.5 \mathrm{mas}$ ). The simulation did not include static non-common path aberrations (NCPA), and WFS noise was simulated at the level of 50mas RMS per subaperture. Results for algorithms $2 \& 3$ (frame-by-frame PSFR) are shown in Figure 5, illustrating accurate reconstruction once all required filters are incorporated. Results for algorithms $4 \& 1$ are shown in Figure 6, showing spurious OTF oscillations for algorithm 4 as observed in Figure 2. Figure 7 summarizes results for the most successful algorithms 1, 2 and 3, which all yield SR and PSF Profile errors of only a few percent. 

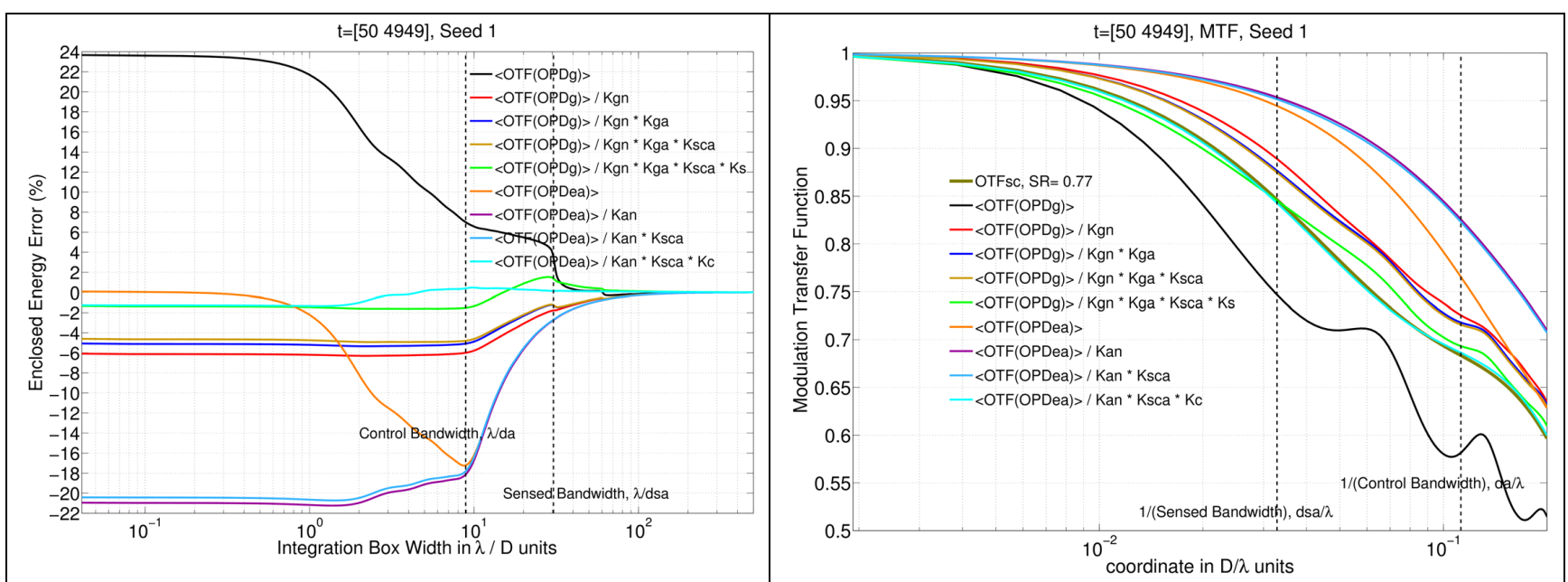

Figure 5: EE errors and OTF cross-sections for algorithms $2 \& 3$.
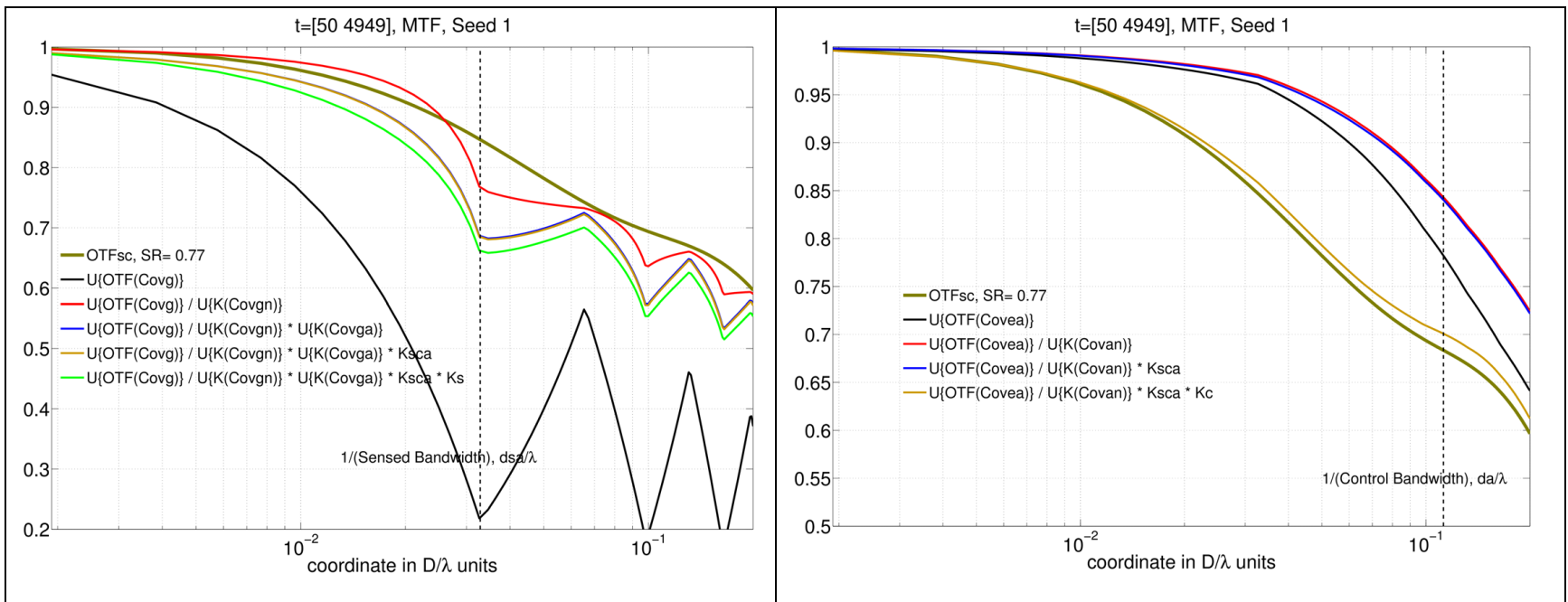

Figure 6: OTF cross-sections for algorithms 4 \& 1.
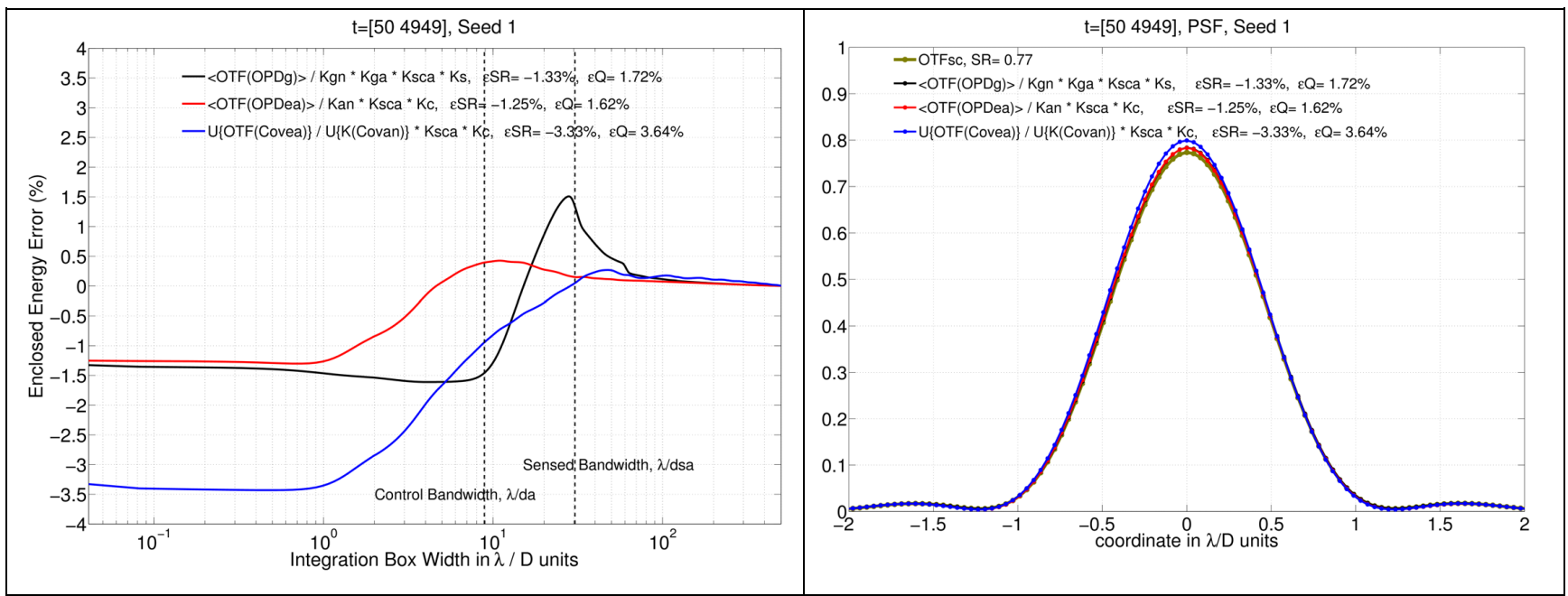

Figure 7: Left: EE errors for algorithms 3 (black), 2 (red), and 1 (blue). Right: reconstructed PSFs. 


\subsection{Results obtained from laboratory data}

The DM IFs were reconstructed from the measured poke matrix (PM) and the scaling factor to convert from a unit DM command to optical path difference (OPD) was determined based upon the fit. Results are illustrated in Figure 8. A single pupil-conjugate rotating phase screen was inserted in the beam, producing a $58 \mathrm{~cm}$ Fried parameter at half micron, and an equivalent wind speed of $15 \mathrm{~m} / \mathrm{s}$. The bench software records an on-axis $122 \times 122 \mathrm{PSF}$ at $670 \mathrm{~nm}$ (5.6mas pixels), exposed during 800 frames (30ms exposures). A dark image is recorded at the beginning of the run for calibration purposes. A static non-common path aberration (NCPA) slope offset is added to the closed loop WFS slopes to provide the best image quality in the absence of turbulence screen. The measured static and turbulence PSFs are displayed in Figure 9.

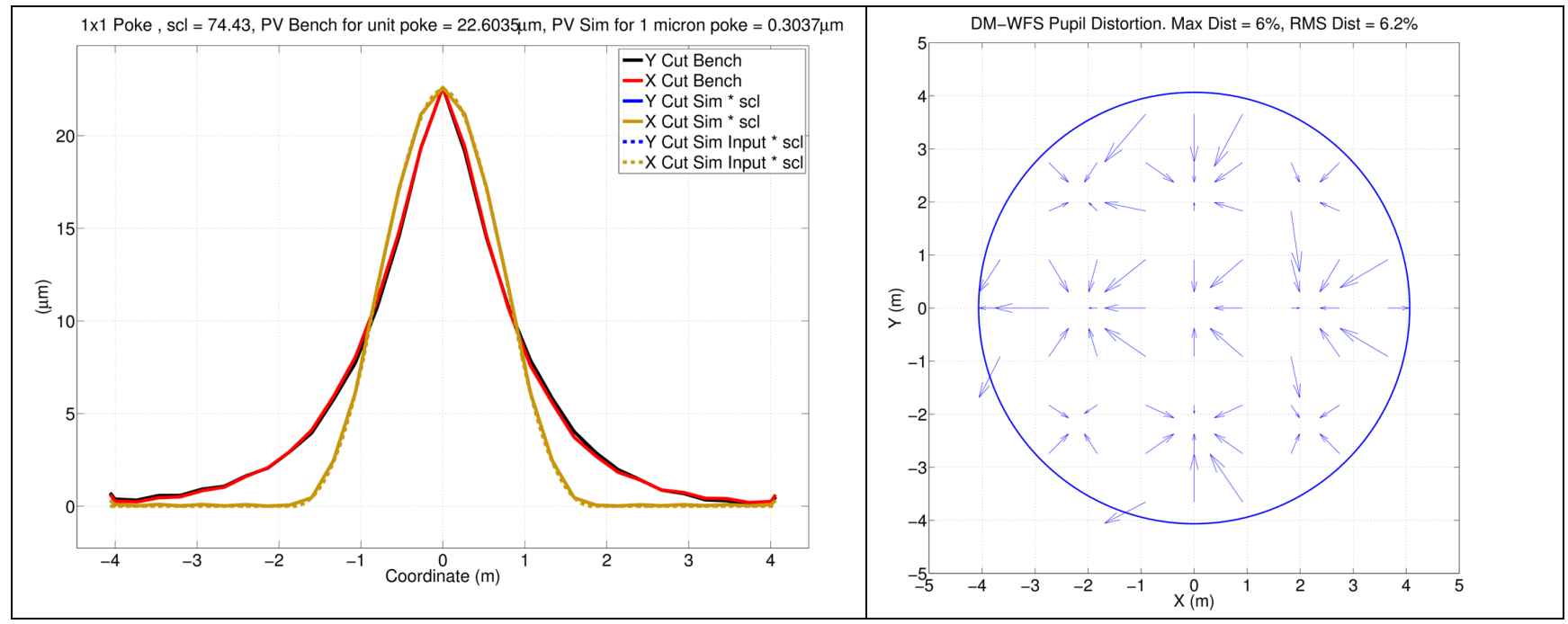

Figure 8: Left: reconstructed actuator unit poke. Right: DM-to-WFS pupil distortion obtained from the peak position of each reconstructed poke.
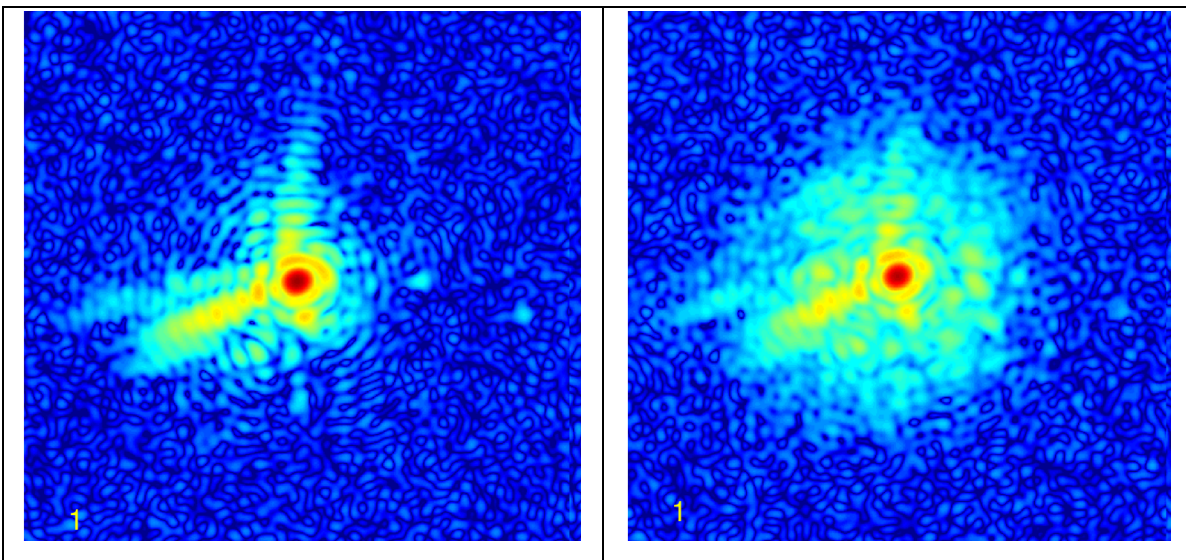

Figure 9: Left: measured closed loop PSF with NCPA slope offsets applied and no phase screen inserted, estimated SR is 64.8\%. Right: measured closed loop PSF with NCPA slope offsets applied and pupil-conjugate phase screen inserted in the beam, estimated SR is 51.2\%. Data recorded on July 072017.

Figure 10 illustrates OTF reconstructions from telemetry data recorded on July 07 2017. Figure 11 shows enclosed energy (EE) error curves as well as cross-sections of the 3 PSF reconstructions. PSFR accuracy is at the level of 5-6\% relative SR error and $8 \%$ PSF Profile error. 


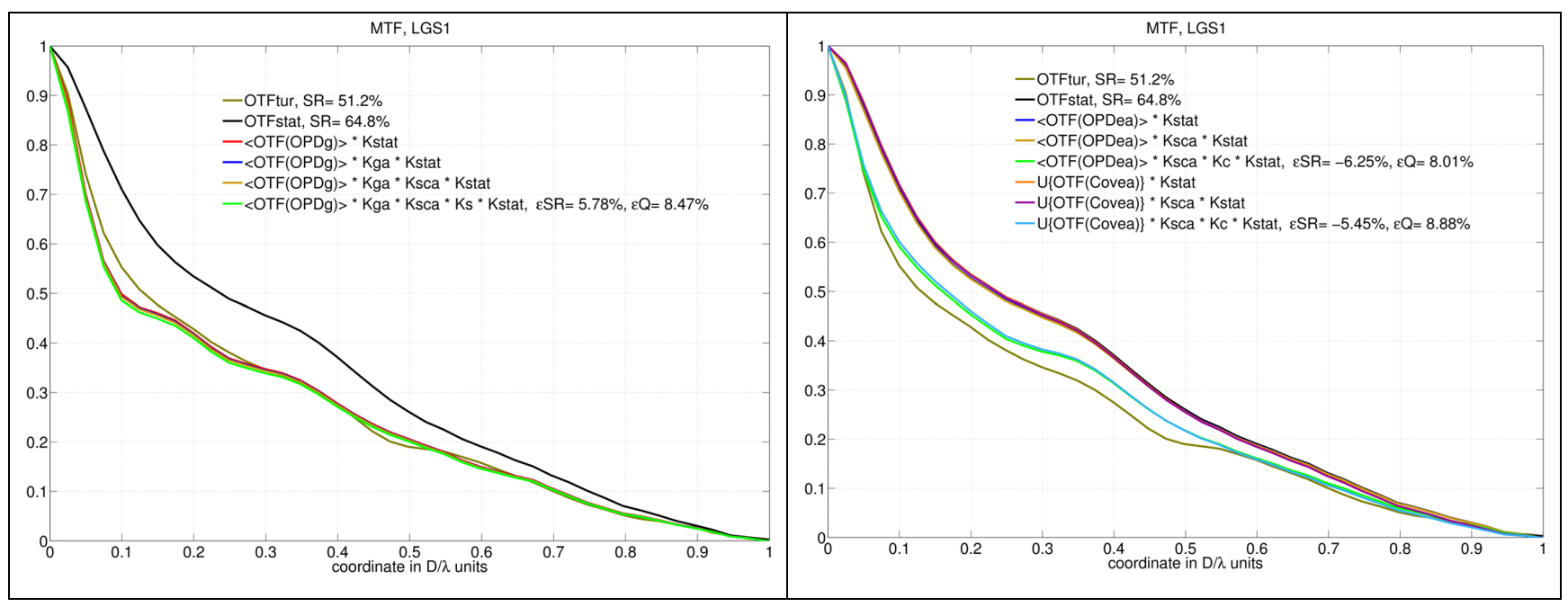

Figure 10: Left: OTF cross-sections from WFS gradient time history (algorithm 3). Right: OTF cross-sections from actuator error time history (Algorithm1) or actuator error covariance matrix (Algorithm2).

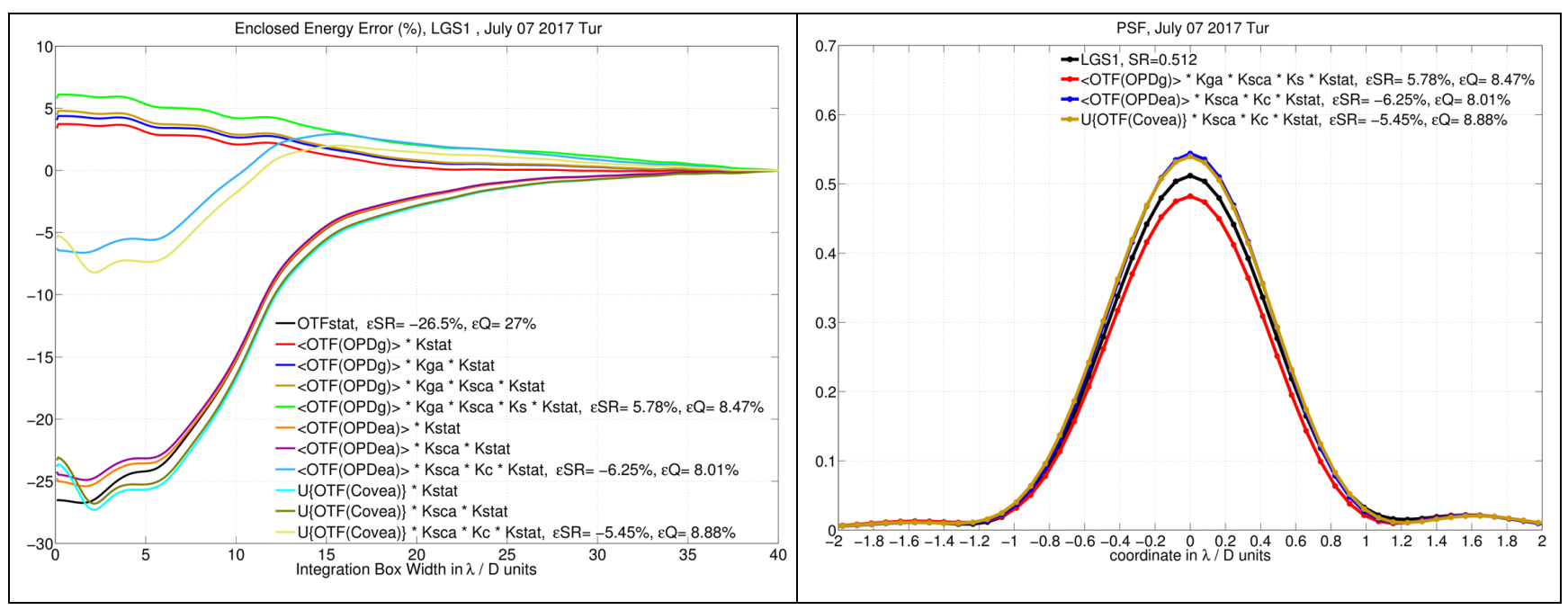

Figure 11: Left: EE error curves. Right: Cross-section of the 3 reconstructed PSFs. The black curve is a cross-section of the measured turbulence PSF displayed on the right panel of Figure 9.

\section{SUMMARY}

We have described 4 algorithms to perform on-axis NGS AO PSFR. We have benchmarked them on simulated telemetry (NFIRAOS and HeNOS) and actual laboratory telemetry acquired on the HeNOS bench. 3 of the 4 algorithms appear to be robust and capable to achieve below $10 \%$ error in SR and PSF Profile. The actuator error based algorithms (Algorithm2 and Algorithm1) appear the most promising since they naturally extend to tomographic PSFR.

\section{ACKNOWLEDGMENT}

The authors gratefully acknowledge the support of the TMT collaborating institutions. They are the Association of Canadian Universities for Research in Astronomy (ACURA), the California Institute of Technology, the University of 
California, the National Astronomical Observatory of Japan, the National Astronomical Observatories of China and their consortium partners, and the Department of Science and Technology of India and their supported institutes. This work was supported as well by the Gordon and Betty Moore Foundation, the Canada Foundation for Innovation, the Ontario Ministry of Research and Innovation, the National Research Council of Canada, the Natural Sciences and Engineering Research Council of Canada, the British Columbia Knowledge Development Fund, the Association of Universities for Research in Astronomy (AURA), the U.S. National Science Foundation and the National Institutes of Natural Sciences of Japan.

\section{REFERENCES}

[1] P.B.Cameron, M.C.Britton and S.R.Kulkarni, "Precision astrometry with adaptive optics," in "Adaptive Optics Systems," N.N.Hubin, C.E.Max and P.L.Wizinowich, eds., Proc. Soc. Photo-Opt. Instrum. Eng. 7015, 70150A-1 70150A-9 (2008).

[2] P.B.Cameron, M.C.Britton and S.R.Kulkarni, "Precision astrometry with adaptive optics," Astron. J. 137, 83-93 (2009).

[3] C.Schödel, "Accurate photometry with adaptive optics in the presence of anisoplanatic effects with sparsely sampled PSF: the Galactic center as an example of a challenging target for accurate AO photometry," Astron. Astrophys. 509, A58 (2010).

[4] R.Davies, H.Engel, E.Hicks, N.F.Schreiber, R.Genzel, L.Tacconi, F.Eisenhauer, S.Rabien, “Dissecting Galaxies with Adaptive Optics," in "Adaptive Optics Systems II," B.L.Ellerbroek, M.Hart, N.Hubin, P.L.wizinowich, eds., Proc. Soc. Photo-Opt. Instrum. Eng. 7736, 7736G-1 - 7736G-9 (2010).

[5] C.Boyer and B.Ellerbroek, "Adaptive Optics Program Update at TMT," Proc. Soc. Photo-Opt. Instrum. Eng. 9909, 990908, doi: 10.1117/12.2232945 (2016).

[6] J.P.Véran F.Rigaut, H.Maitre and D.Rouan, “Estimation of the adaptive optics long-exposure point-spread function using control loop data," J. Opt. Soc. Am. A 14, 3057-3069 (1997).

[7] M.C.Britton,"The anisoplanatic point-spread function in adaptive optics," Pub. Astron. Soc. Pac. 118, 885-900 (2006).

[8] R.Flicker,"PSF reconstruction for Keck AO," W.M.Keck Observatory, Kamuela, Hawaii 96743, USA (unpublished, 2008). Available at http://www.oir.caltech.edu/twiki_oir/bin/view/Keck/NGAO/PSF

[9] S.Ragland, L.Jolissaint, P.Wizinowich, M.van Dam, L.Mugnier, A.Bouxin, J.Chock, S.Kwok, J.Mader, G.Witzel, T.Do, M.Fitzgerald, A. Ghez, J.Lu, G.Martinez, M.R.Morris and B.Sitarski, "Point spread function determination for Keck adaptive optics," Proc. Soc. Photo-Opt. Instrum. Eng. 9909, 99091P, doi: 10.1117/12.2231814 (2016).

[10] B.Ellerbroek and D.Tyler, "Modeling the combined effect of static and varying phase distortions on the performance of adaptive optical systems," Appl. Opt. 38, 3857-3868 (1999).

[11] M.Rosensteiner, P.Turri, E.Mieda, J.P.Veram, D.Andersen and G.Herriot, "On the verification of NFIRAOS algorithms and performance on the HeNOS bench," Proc. Soc. Photo-Opt. Instrum. Eng. 9909, 990949, doi: 10.1117/12.2233054 (2016). 